\title{
A step-by-step approach for science communication practitioners: a design perspective
}

\author{
Maarten C.A. van der Sanden, Frans J. Meijman
}

\begin{abstract}
Science communication processes are complex and uncertain. Designing and managing these processes using a step-by-step approach, allows those with science communication responsibility to manoeuvre between moral or normative issues, practical experiences, empirical data and theoretical foundations. The tool described in this study is an evidence-based questionnaire, tested in practice for feasibility. The key element of this decision aid is a challenge to the science communication practitioners to reflect on their attitudes, knowledge, reasoning and decision-making in a step-by-step manner to question the aim, function and impact of each issue and attendant communication process or strategy. This approach eventually leads to more professional science communication processes by systematic design. The Design-Based Research (DBR) derived from science education and applied in this study, may form a new methodology for further exploration of the gap between theory and practice in science communication and. Practitioners, scholars, and researchers all participate actively in DBR.
\end{abstract}

\section{Context and objectives}

Bubela et al. ${ }^{1}$ write in Nature Biotechnology that science communication becomes increasingly complex due to science and technology's development and intricate relationship with society. Science-related controversies in society typically involve clashes of values and beliefs. These controversies are due not only to deficits of scientific understanding, but also of social capital. ${ }^{2}$ The deficits not only increase the complexity of the controversies, but the uncertainty of processes of science communication as well. The medical field teaches us that, particularly in the public arena, mono-causal explanations and simplistic solutions ignore the complexity of the relationships between individual health, care and public health. Values, norms and conventions in these three domains may be discordant. ${ }^{3}$ Therefore, for the practice of science communication, we consider creating balances within the individual and between individuals, society and the scientific and professional domains as a basic assumption.

However, the gap between science communication theory and practice is a deficit of the science communication domain itself. At the $\mathrm{PCST}^{4}$ conferences where practitioners, scholars and researchers gather from all over the world, one recognizes a wide field of practice and a much smaller field of theory that in practice is rarely integrated. This problem is also often mentioned in science communication textbooks. ${ }^{5,6,7}$ Moreover, practice is multifaceted ranging from well equipped, experienced or at least fulltime information officers, science writers, web designers or science journalists, to practitioners, managers or researchers who are only occasionally involved in science communication. This study examines a science communication officer working in an academic institute for gene technology and society, who needs to communicate about new developments in predictive DNA-diagnostics. How should he prepare for various meetings with disparate audiences?

To overcome the gap between theory and practice while addressing various people involved in or with science communication, we argue that a systematic approach to facilitate the design perspective of science communication professionals might be helpful. This design perspective allows the professional to cope with a science communication problem efficiently and effectively, while taking into account the aforementioned contextual constructs and variables; thus leading to an optimised science communication process. How can this approach prove to be insightful and manageable for the science communication practitioner looking for a profound and sustainable communication process? How can science 
communication practitioners be challenged to combine theory and practice, even theories that are not directly related to science communication? How can science communication practitioners or professionals improve their competence and accountability? To address these questions, this article focuses on:

1) the relevance of designing science communication processes and approaches to professional decision-making;

2) a description of a validated questionnaire, and its relevance for challenging practitioners (to provoke reactions);

3) Design-Based Research (DBR) bridging theory and practice in science communication by continuous interaction with practitioners leading to a manageable practice and reflection on theory. ${ }^{8}$

\section{Relevance of design in complex professional decision-making}

From a design perspective of changing existing situations into preferred ones, ${ }^{9}$ the professionally competent practitioner constructs a relevant combination of the social, contextual, structural and outcome variables of the science communication process. As in a product design process, the designer combines variables such as usability, aesthetics and sustainability into a product that fits the beliefs, emotions, and wishes of the consumer and at the same time is producible and saleable. Product design is all about optimisation and alignment of technology, context, product, production and market. The same holds for science communication, as mentioned in this study, where DNA-technology, medical context, commercially available kits and the market are facets of the communication process design. The design perspective helps practitioners who are making decisions in an ill-structured design process and who are experiencing science communication problems move to optimised processes.

In practice, science communication practitioners often make numerous decisions based on tacit knowledge, experiences, intuition, implicit theoretical reasoning and available data. This may lead to success or failure. However, in both cases it is hard to understand the reason for success or failure using hindsight, because it is not clear how the variables in the process worked together. These explicit insights are necessary for further development and optimisation of an effective science communication process.

To obtain these insights, one can conduct evaluation research; however, evaluations take time and money and can lead to a delay in further development of a project in a changing and moving society. The design perspective relocates the effort of identifying problems to a point in the process before the problems have arisen, whilst neither delaying the communication process nor interaction with the target audiences. A design approach is generally based on a design methodology ${ }^{10}$ and results in a sequence of steps:

1) problem analysis leads to design criteria;

2) synthesis leads to a preliminary design;

3) simulation leads to insight into expected properties;

4) evaluation of design leads to adjustments of science communication processes.

Moreover, we have added the explicit use of (science) communication theories, as well as theories from other domains, in step 1 and 2 connected in a so-called theoretical framework.

Some typical characteristics of the steps above are as follows. First, a design-oriented practitioner broadens the problem, creating a greater problem and solution space ${ }^{11}$ that may lead to new insights and solutions, whereas the inexperienced practitioner looks only for a single solution. ${ }^{12}$ Second, a design process is about making essential choices explicit. Third, in broadening the problem and to synthesis a solution, it is necessary to create a theoretical framework by connecting (science) communication theories on conceptual, theoretical, model, construct or variable levels. Such a theoretical framework might contain 10 or 20 distinct concepts and theories with attendant constructs and variables. The creation of this framework raises questions and rethinking about the science communication process and the practical criteria and implications for science communication professionals. Therefore, the theoretical framework combines theory and practice. In professional design there is a continuous back and forth movement between problems and solutions. ${ }^{12} \mathrm{~A}$ decision aid for science communication practitioners should support this process of step-by-step, forward-backward, explication, elicitation (see below) and decision-making. But what does making decisions theoretically mean? 


\section{Decision-making}

Decision-making arises from the need to select the best possible course of action (or a set of optimized actions) from a set of alternatives. ${ }^{13}$ On a process level, decision-making is a knowledge-intensive activity with knowledge as a raw material, work-in-progress, by-products, and finished goods. ${ }^{14}$ However, generally there are data missing and there is no clear vision on where to proceed. We reckon the decision-making problem in science communication is complex, for two or more of the following conditions apply simultaneously. ${ }^{15}$ First, the actor pursues several objectives simultaneously, although some objectives are not precisely defined and it is possible that contradictions exist. Second, many levels of control or decision variables exist to reduce the gap between the target and the existing situation. However, decision variables have several possible features. Thus, the levels of control or decision variables, as well as the possible features of the latter, lead to a large number of possible problem-solving options. Third, the future development of several environmental variables cannot be predicted with certainty. Therefore, the actor has to evaluate problem-solving options based on several possible environmental scenarios. Finally, the actor possesses only limited experience or models to determine the consequences of the available options, in part due to these three described conditions.

Contrary to simple decision-making problems that are always choice problems and often meet the requirements of a well-structured decision problem, complex problems (as in the case of this article) are always ill-structured. Therefore, design problems can usually be tackled as described above. To do so, one may need a decision aid to reduce the complexity and uncertainty. In the case of clinical decisionsupport systems practitioners are primarily motivated to use these systems when this technology allows them to forecast the potential outcomes of decisions, prior to actually making those decisions. ${ }^{16}$

\section{Elicitation}

Another important aspect of decision-making is challenging the decision maker. For problem solving and solution finding, users are generally unable to identify preferences beyond basic ones. ${ }^{17,18} \mathrm{Pu}$ states that professionals are able to critique given suggestions and solutions and this eventually makes decisions more tangible and understandable. Therefore, decision-support systems should support rather than automate (take over) decision making. ${ }^{17,19}$ Design systems provoke reactions and explications, encourage discussions, reduce problem complexity and encourage consideration of alternatives, ${ }^{17,19}$ in other words, elicit practitioners. According to Heinrichs, ${ }^{17}$ a decision-support system should encourage expert design methods such as: search for information, critiquing examples and making trade-offs at a structural level. We have attempted to develop and test a decision aid for biomedical science communication on predictive DNA diagnostics ${ }^{20,21}$ making use of design thinking and design methodology.

\section{Theoretical framework}

To develop the decision aid we started with Miller and Kimmel's ${ }^{22}$ model of biomedical science communication. Their model describes how an individual is influenced by peers, spouse, media and education, and how all these influences are factually and emotionally important for the individual. Their theory is based on multiple surveys that generate insight into what individuals consider as the most important sources for information. For our case, we conclude that the model needed to be expanded, for example, in consideration of the basic notions and prejudices an audience might have on the subject of DNA in general, or specifically on predictive DNA testing. Thus, we expanded the theoretical framework from theories connected to various areas of communication. Our focus was on identified variables that determine the success or failure of communication processes within health communication, medical psychology and (medical) commercial communication (advertising). Communication variables were systematically selected from the literature regarding structure, process, outcome and context as communication evaluation criteria. ${ }^{23}$ 


\section{Predictive DNA-diagnostics: a case}

Communication on predictive DNA diagnostics is complex on informational and communicational levels. One might ask: how much information about genetic screening or other use of DNA-diagnostics do audiences need? And what claims can be made? In light of informed consent, it is important that the public has a realistic view of the potential and expectations for this technology. In the United States, a similar discussion focused on the issue of the commercial availability of genetic tests. ${ }^{24}$

These discussions do not begin just from providing information. For example, in the case of breast cancer screening, a first step could be asking the lay audience what they want to know, instead of immediately giving them the information, not to mention instruction. ${ }^{25}$ Therefore, lay-centred systems such as those provided by DISCERN ${ }^{26}$ and $\mathrm{ACCE}^{27}$ can be used to appraise information quality, but this information does not necessarily fit the target audience or develop a social basis in terms of trust, credibility or convincing power. The lay audience may need to know how to search for information and to mobilize and discuss the diverse values and emotions connected to this issue. For a person active in the field of science communication, it is hard to keep track of all the aspects of predictive DNA-diagnostics and attendant societal, organizational, political and individual values at one time. DNA-technology, research and emotions are intertwined. ${ }^{28,29}$

\section{Decision aid}

The decision aid (a questionnaire, step-by-step plan) we developed consists of three distinct phases: 1) analysis - target audiences' views and expectations, and the determination of the content and style of the message; 2) synthesis - the determination of the communication objectives and goals; 3) determination of the communication strategy and intervention - how to communicate with whom and when? The following questions are answered:

1. with whom am I going to communicate on which subject and what risk of miscommunication exists?;

2. what is the theoretical frame and concluding message from which I am going to start my communication with the selected target audience(s) and what effects do I expect?;

3. how am I going to communicate with my target audiences to achieve the expected effects based on the theoretical frame and process criteria obtained from the analyses of the target audience, aim and message?

The questionnaire included 13 questions with 49 possible responses. In phase 1 of the creation of the decision aid (analysis), we developed questions on the presumed notions, perceptions and predispositions of the various segments of the target audiences. These questions were to be answered by the science communication practitioner. The practitioner had to also explicitly consider an estimation of the risk of miscommunication if necessary information could not be included in the analysis.

Another question within phase 1 investigates the emotional distance between the target audience and the object of communication. If an individual is a patient, they probably already have a stronger emotional involvement with the subject of predictive DNA diagnostics, compared to a citizen who has just learned about the scientific possibilities of predictive DNA diagnostics. Emotional distance is further elaborated in variables such as the (ir)reversibility and perceived seriousness of the disease or health-threat. Phase 1 concludes with a question on the level of the need for knowledge (factual, moral, ethical, emotional) of the target audience based on the constructs and variables previously asked, and in addition to the estimation of the risk for miscommunication.

In phase 2 (synthesis), questions are asked about distinct modalities of science communication like science promotion, science education or the prevention of knowledge deprivation. ${ }^{21}$ Questions are also presented about the aims of science communication, such as public understanding of science, public awareness, public engagement and public participation. ${ }^{29}$ The science communication practitioner needs to make explicit choices establishing a coherent relationship between modality (science promotion, science education and prevention of knowledge deprivation), ${ }^{21}$ aim and the intended effect of the science communication process (public awareness of science, public understanding of science, public engagement of science and public participation in science). ${ }^{22}$ Phase 2 ends with a description of the message based on the target audience's specific need for knowledge (phase 1) connected to a coherent chain of modality, aim and effect. 
In the case of predictive DNA diagnostics, the process may start from the point of view of science promotion in which the role and impact of genetic research is discussed, whilst the main science communication aim could be public awareness of science.

The decision aid again asks the practitioner to assess the level of uncertainty. Corresponding to questions in phase 1, the practitioner is asked if the message developed actually suits the beliefs and aims of the target audiences (and on what level), or if there is a risk of a certain level of miscommunication. In phase 3 , the intervention is determined using the results of phase 1 and 2 . The communication strategy that the communication professional applies, based on a specific theoretical frame (e.g., the Health Belief Model $^{30}$ or the Elaboration Likelihood Model $^{31}$ ) establishes the coherence between modality, aim, strategy and means of communication.

By proceeding through all three phases, the communication practitioner has an explicit description of the problem, optional ideas for the intervention (communication strategy), a view on the expected outcomes and an estimation of the risks for miscommunication. Answering the questionnaires and being challenged to write down ideas, thoughts and decisions stimulates professional science communication thinking and makes decisions explicit. This outcome reaches further than existing checklists, protocols and fixed frames of communication strategies as reported by the respondents in this study described in the following section.

\section{Evaluation, methodology and experiment}

We conducted a study using the decision aid with (science) communication practitioners and policymakers working with predictive DNA diagnostics. The participants were requested to reflect on a fictive case study about lung cancer and predictive DNA diagnostics.

An interviewer conducted 10 qualitative interviews with three communication practitioners, two representatives of patient organisations, three research and executive specialists and two policy makers and strategy officers. The reflective process had three phases: 1) individual completion of the questionnaire based on a fictive case study about a predictive test for lung cancer, 2) an interview conducted by an interviewer with questions about the circumstances and reasons for the answers given on the questionnaire (how, why, was the questionnaire clear, were there elements that should be added), and 3) a group meeting in which the main conclusions were discussed once again. Thus the prototype decision aid was discussed 21 times at various stages of development. Below, we present some examples of questions and reflection possibilities, based on the answers of one of the respondents, a science communication officer at an academic institute for gene-technology and society. The answers of the respondent are in italics.

Phase 1: Question 1: Which notions and beliefs exist within your target audience?

\begin{tabular}{|l|l|}
\hline 1.1 Target audience's attitude towards technology: & \\
\hline Technology and science are a panacea for all problems & \\
\hline Technology and science should not be trusted & \\
\hline Technology and science will end up in a future shock & $X$ \\
\hline Technology and science mean prosperity & \\
\hline Technology and science should be embraced & $X$ \\
\hline Technology and science are assimilated in society & \\
\hline People should adapt to technology and science & \\
\hline
\end{tabular}

\begin{tabular}{|l|l|}
\hline 1.2 Cultural themes connected to health and disease: & \\
\hline People should be cured on a natural basis & $X$ \\
\hline People should be cured on pharmaceutical basis & $X$ \\
\hline People are strongly attached to traditions & \\
\hline My target audience is open to changes & $X$ \\
\hline My target audience thinks along dichotomies: health/disease, wrong/right, & \\
\hline
\end{tabular}


Describe your target audience by making use of the insights obtained above:

A: My target audience are people who find that: Science could solve many things. In the meantime most people in the target audience do know that lifestyle and coincidence (bad luck?) determine if you will be healthy or not. Another part of the target audience searches for help with natural medicine.

Risk 1: If you are not able to determine the prejudices and basic notions of the target audience you may risk miscommunication. Describe the risk you think you have taken.

Risk 1: Risk for my target audience is that they might have a healthy lifestyle and therefore do not see any risk for lung cancer.

During the questionnaire, the descriptions of the target audiences, their aims and how to fit communication to those aims are continuously and cumulatively addressed. For example:

The answers to the first six questions (block A and B together with question 5 and 6) lead to a description of the target audience including their proto-knowledge and how they use this knowledge. Describe in block C your target audience extensively.

C: My target audience are people that: want to choose for themselves. They are not easily convinced or willing to take part in various programmes. Their knowledge is reasonably profound and that steers their willingness for receiving new information. Chance is reasonably big that even the smokers in this target audience are not going to participate in a national health program.

Risk 6: If you take your answers so far into consideration what then will be the risk for miscommunication?

Risk 6: Risk is that people do think that the program is meant for smokers only. However the screening program is meant for everyone. Moreover, a genetic test is not easily understandable from the risk point of view.

Phase 1 ends in the description of a core message:

Now it is possible by using the answers to all the questions in phase 1 (block A, B, C and D and the question 7 and 8) to describe the main message and the overall estimated risk for miscommunication.

E: taking all the above into account, could you write down the main message: smoking is not the only reason for lung cancer. The target audience should have a clear idea about why and how to obtain such a test. For women I might make a connection with the communication on breast cancer.

$\mathrm{F}$ : describe the overall estimated risk for miscommunication. In what way is the effect of the content of your message certain/uncertain?: relationship between lung cancer and smoking is obvious and known. Therefore, it might be difficult for the target audience that they are not off the hook while they don't smoke. There might be a hereditary risk. Smokers on the other hand might find an excuse in this message on possible genetic causes, to keep up smoking. I want to raise the sense of urgency for both smokers and non-smokers that might be difficult and raises questions from the target audience. 
Finally, in phase 3 there is a summary (respondent chose the Health Belief Model and focused on a dialogue about the facts of lung cancer):

I: I've chosen this strategy: because the target audience first should be sensitized to have some knowledge. This could be achieved by cognitive dissonance. "You do know a lot about lung cancer, but did you know about the hereditary component? Even if you do not smoke there is a chance."

Risk 13: aim, message, intervention and strategy should be aligned. Describe the risk for miscommunication: A part of the target audience need, despite their own knowledge and experiences, to be told what exactly needs to be done. Decide whether to take the test or not is too insecure.

Here one can see that using a theory and reasoning on risks for miscommunication, the respondent focused his message and strategy for the outcome of phase 2 .

During the group meeting, the respondents came to the conclusion, individually and collectively, that the questionnaire makes sense for, and is of use to practitioners who have to think in highly abstract terms about the social design of biomedical communication activities, and new activities in particular. The respondents feel that the theoretical framework and the decision aid provide insight into the communication process making it manageable and possibly effective for the future. The aid helps practitioners to ask questions and to take a critical view of the process. Remarks for improvement were formulated as follows:

The underlying theoretical framework and questionnaire for practice could be used by practitioners in complex biomedical science communication cases in which the model and questionnaire are needed to support reflection on the design of a biomedical science communication process at the policy and strategy level. Questions referring to ethical constraints should be added, as well as other, different models, and theories like ELM and HBM.

This test supports the idea of a gap between communication theory and practice in which there are differences among researchers, policy makers and communication practitioners. A translation from theory to practice, one which is optimally flexible for different user groups, is needed. This questionnaire is a first step for the practice of biomedical science communication but needs to be refined. Therefore, the underlying model and questionnaire should be used as a platform for further development of practice and theory. Ethical constraints are one example of an issue that should be refined and made manageable for practice on distinct levels.

Most important is the idea that the questionnaire functions as a platform for testing new variables, the sequence of questioning, and, a rhetorical ground for better understanding between researchers and practitioners. The questionnaire can be considered as a kind of selfreferential system.

\section{Discussion and conclusion}

The aid we developed does not prescribe professional thinking but rather provides support by raising questions. So this decision aid is meant as a field guide, not as a guideline. A practical field guide for actually binding theory and practice and making communication processes more understandable, manageable, tangible and therefore elegant by design. Within the design of communication processes, theoretical possibilities and practical boundaries meet, such as partial knowledge of the target audiences or a tight time frame and budget. Of course, heuristics (i.e. rules of thumb) guide practitioners to the direction of a solution too. However, such a heuristic does not guarantee the high quality or practical feasibility of that solution. ${ }^{9}$ Moreover, the decision aid was only tested by 10 respondents in 20 interviews and 1 group meeting. As such, this recent research is a first step in further developing decision aids for science communication professionals to improve and increase their level of professionalism by continuously reflecting on their decisions. 


\section{Design-Based Research (DBR)}

By designing (communication) processes and instruments, both researchers and practitioners recognise theoretical blind spots from a practical point of view and practical blind spots from a theoretical point of view. The latter is an important characteristic of the research methodology of DBR, which aims to reflect on theory as well as on practice by performing a real intervention in practice using evidence-based prototypes such as the discussed decision aid. DBR involves practitioner participation, which is another important aspect. Theory and practice are intertwined in a sequential evaluation and adjustment iterations in which researchers and practitioners work together, for example, with focus groups, observations and workshops. Generally, biomedical science communication practitioners implicitly use a set of choices and criteria, considering a 'best' fit to their perceived practice. By making these choices more explicit in a design aid, the communication practice becomes more transparent. Then practitioners become more professional are able to first point out the intertwined moral and normative issues, practical experiences, empirical data and theoretical foundations. Second, they can then design communication processes on the basis of these issues by systematically questioning the aim, function and impact of each issue. From the idea that there is no silver bullet for an ultimately effective science communication process, providing a design aid for this process should make use of the best possible theories and available information, thus ensuring process optimisation.

Linking theory to practice and vice versa combines moral and normative issues, practical experiences, empirical data and theoretical foundations. If one of these elements is missing, the outcome of a science communication process will probably be less effective. However, in an efficiently executed science communication practice, the science communication professional has to make decisions since not all elements can be included equally (and do not need to be included) in the science communication process. An inclusive and manageable decision aid as we propose, supports the practitioner in designing this optimised and effective, manageable, communication process by means of elicitation.

Moreover, really linking theory and practice requires the interaction and participation of both the professional and the researcher. DBR provides this link by developing and testing prototypes in real practice such as our decision aid. Considering effective science communication as a design problem of a social process enriches and enhances both the practice and theory of the best possible effective science communication. Decision aids function as a platform for integration and reflection on science communication.

\section{Acknowledgements}

The authors wish to thank the reviewers for their profound comments and suggestions for improvement of this manuscript.

\section{Note and references}

T. Bubela et al. (2009), Science communication reconsidered, Nature Biotechnology, 27(6): 514-518.

S. Hornig Priest (2008), Linking theory and practice, Science Communication 30(1): 5-7.

F.J. Meijman (2008), Health communication and public media: professionals need to be heard, Nederlands Tijdschrift voor Geneeskunde 152: 1760-1764.

4 PCST: International Network on Public Communication of Science and Technology.

5 D. Cheng et al. (eds.) (2008), Communicating science in social contexts. New models, new practices, Springer, Germany.

${ }^{6}$ M. Bucchi and B. Trench (eds.) (2008), Handbook of public communication of science and technology, Routledge, New York U.S.A.

7 R. Holliman, E. Whitelegg, E. Scanlon, S. Smidt and J. Thomas (eds) (2009), Vol. I: Investigating science communication in the information age, Oxford University Press, Oxford U.K.

8 E. Baumgarter et al. (2003), Design-based research. An emerging paradigm for educational inquiry, Educational Researcher, 32(1): 5-8.

9 P. Kroes, A. Light, S.A. Moore and P.E. Vermaas (2008), Design in engineering and architecture: towards an integrated philosophical understanding, in P.E. Vermaas et al. (eds.), Philosophy and design from engineering to architecture, Springer Germany.

${ }^{10}$ N.F.M. Roozenburg and J. Eekels (2003), Productontwerpen, structuur en methoden, Uitgeverij Lemma, Utrecht The Neatherlands. 
${ }^{11}$ C. Ho (2001), Some phenomena of problem decomposition strategy for design thinking: differences between novices and experts, Design Studies 22: 27-45.

${ }^{12}$ N. Cross, Expertise in design: an overview, Design Studies 25(5): 427-441.

${ }^{13}$ L.C. Jain and C.P. Lim (2009), Advances in decision making, in E. Rakus-Andersson, R.R. Yager, N. Ichelkaranje and L.C. Jain (eds.), Recent advances in decision making (pp. 1-6), Springer Verlag, Berlin Germany.

${ }^{14}$ R. Bose (2003), Knowledge management-enabled health care management systems: capabilities, infrastructure, and decision support, Expert Systems with Applications 24: 59-71.

${ }^{15}$ R. Grünig and R. Kühn (2009), Successful decision-making. A systematic approach to complex problems, Springer, Heidelberg Germany.

${ }^{16}$ S. Weber, K.L. Courtney and M. Benham-Hutchins (2009), Decision support in multi-professional communication, Journal of Medical Systems 33: 59-65.

${ }^{17}$ R. Heinrichs (2011), A literature survey on the employability of decision support systems while designing science communication strategies, Unpublished literature review, Master thesis, Delft University of Technology.

${ }^{18} \mathrm{P}$. Pu, B. Faltings and M. Torrens (2003), User-involved preference elicitation, in IJCAI Workshop on Configuration, pages 56-63.

${ }^{19}$ F. Burstein, C. Holsapple, D. Arnott and G. Dodson (2008), Decision support systems failure, in Handbook on Decision Support Systems 1, International Handbooks Information System, pages 763-790, Springer Berlin Heidelberg, Germany.

${ }^{20}$ M.C.A. Van Der Sanden and F.J. Meijman (2008), Dialogue guides awareness and understanding of science. An essay on different goals of dialogue leading to different science communication approaches, Public Understanding of Science 17(1): 89-103.

${ }^{21}$ M.C.A. Van Der Sanden (2008), Towards effective biomedical science communication. A composit theoretical framework making biomedical science communication on predictive DNA diagnostics understandable and manageable, Thesis, Vrije Universiteit Amsterdam, The Neatherlands.

22 J.D. Miller and L.G. Kimmel (2001), Biomedical communications. Purposes, audiences and strategies, Academic Press, San Diego U.S.A.

${ }^{23}$ A. Donabedian (1980), Methods for deriving criteria for assessing the quality of medical care, Medical Care Review 37(7): 653-698.

${ }^{24}$ S. Parthasarathy (2007), Building genetic medicine. Breast cancer, technology, and the comparative politics in health care, MIT Press, Cambridge, Massachusetts U.S.A.

${ }^{25}$ H. Van Maanen (2002), Medische publieksvoorlichting in de praktijk: de uitnodiging voor bevolkingsonderzoek, in F.J. Meijman and F. Meulenberg (eds.), Medische publiekscommunicatie. Een panorama, Bohm Stafleu van Loghum, Houten.

${ }^{26}$ DISCERN as the website describes has been developed to ensure that there is an accessible appraisal tool for patients and other users of information about genetic screening and testing, and that information providers and producers have access to wellresearched guidelines (http://www.discern-genetics.org).

${ }^{27}$ ACCE takes its name from the four components of evaluation: analytic validity, clinical validity, clinical utility and associated ethical, legal and social implications. It is a process model for evaluating data on emerging genetic tests (http://www.cdc.gov/genomics/gtesting/acce.htm).

${ }^{28}$ T.M. Marteau and M. Richards (eds.) (1996), The troubled helix. Social and psychological implications of the new human genetics, Cambridge University Press, Cambridge U.K.

29 A. Van der Auweraert and C.J. van Woerkun (2003), Een queeste (speurtocht) naar een gemeenschappelijk denk-en handelingskader voor wetenschapscommunicatie, in M.L. Noorlander et al. (eds.), Proceedings Kennisdagen 2003: effectieve publiekscommunicatie: hints voor wetenschaps- en techniekcommunicatie (WTC), Report Stichting Weten, Amsterdam The Neatherlands.

${ }^{30}$ N.K. Janz and M. Becker (1984), The health belief model a decade later, Health Education Quaterly 11(1): 1-47.

${ }^{31}$ R.E. Petty and J.T. Cacioppo (1986), From communication and persuasion. Central and peripheral routes to attitude change, Springer Verlag, New York U.SA.

\section{Authors}

Maarten C.A. van der Sanden is an assistant professor in science communication at the Delft University of Technology in Delft in the Netherlands. His research and education focuses on the design of science communication process and the development of corresponding science communication decision aids. In his research, the complexity of the science communication process and bridging the gap between theory and practice are a Leitmotiv. E-mail: m.c.a.vandersanden@tudelft.nl.

Frans J. Meijman is an associate professor of medical science communication, journalism and journalism history at the VU university medical center in Amsterdam in the Netherlands. His research on medical science communication includes the communication process in a broad sense in present and past times, particularly focused on the interaction of health, media and the public domain.

E-mail: fj.meijman@vumc.nl.

How to CITE: M.C.A. van der Sanden and F.J. Meijman, A step-by-step approach for science communication practitioners: a design perspective, Jcom 11(02) (2012) A03. 